\title{
Editorial
}

\section{Reducing socioeconomic inequalities in road traffic injuries: time for a policy agenda}

The existence of differences in the impact of injuries among socioeconomic groups has been well documented, similarly to what is known for most health outcomes. For the case of paediatric injuries, as well as for domestic injuries, variables such as poverty, low socioeconomic position, less privileged social class, ethnic group, urban deprivation, low educational level, and unfavourable family context have shown to be associated with a higher risk of injury morbidity and mortality.

This relation has been less explored for the case of road traffic injuries (RTIs), especially as regards young people. This is most important when keeping in mind the highly negative prospects regarding the expected health impact of RTIs in the world, predicted to be the second leading cause of years of life lost and the third leading cause of disability adjusted life years in 2020. ${ }^{1}$ Moreover, little evidence is available when looking at the impact of socioeconomic inequalities among the different modes of transportation. This is especially relevant, because the last quarter of the 20 th century has seen a large increase in the diversity of transportation modes, especially two wheel motor vehicles, such as mopeds and motorcycles.

A paper by Hasselberg et $a l^{2}$ in this issue provides new evidence on the existence of substantial differences in RTIs during childhood and youth between socioeconomic groups in the Swedish population. Such differences are present as much among pedestrians as among cyclists, moped riders, motorcyclists and car drivers, and except for cyclists, the differences remain after taking into account gender. Moreover, the authors most pertinently estimate that population attributable risks - that is, the percentage reductions in the overall injury rates that would have occurred if all persons experienced the rates of the highest socioeconomic group - would be of more than one third for motorcyclists and car drivers and of one quarter for moped users.

Whether such socioeconomic gaps reflect differences in exposure to traffic, in risk factors or in the severity of the outcome, remains to be clarified. Still, these results underscore the need to consider socioeconomic inequities when designing policy interventions in the field of RTI prevention. Such an approach is generally lacking in most policy formulations. A review of some of the key documents in this field ${ }^{3}$ shows that, in most instances, no references are made to the non-random distribution of road injuries among social groups; consequently, little effort is devoted to intervention strategies aiming at the reduction of these unfavourable social differentials.

In the area of motor vehicle injury prevention, the Haddon matrix has proved to be a useful conceptual tool to apply basic principles of public health to traffic safety, ${ }^{4}$ later extended to other types of injuries. This framework proposes a four column by three row table, where the columns are interacting factors that contribute to the injury process (grouped under host, agent/vehicle, physical environment and social environment factors), while the rows refer to the phases at which change would have its effect, either pre-event, event or post-event (pre-crash, crash or post-crash in its original formulation). This enables the identification of a wide range of interventions that fit within each cell of the matrix, and therefore help orient strategies to be implemented. Acknowledging the need to incorporate the use of value criteria in the decision making process, Runyan ${ }^{5}$ has proposed to include a third dimension to the matrix, suggesting the use of standard criteria such as effectiveness, cost, freedom, equity, stigmatisation, preferences, and feasibility.

Despite such an innovative and potentially useful proposal, we are not aware of an explicit use of such approach in current policy formulations in the area of RTI prevention. Consequently, the consideration of equity as a decision criteria remains to be introduced in the planning process in most settings. This is most relevant as regards vertical equity, that is, in Runyan's terms, the unequal treatment of unequally situated individuals so as to make them more equal with respect to a particular attribute, such as injury risk. In policy terms, this largely means applying interventions to "narrow the gap" between high and low level socioeconomic groups in the differential impact of injuries.

Although ideological and political considerations are obvious determinants of the degree of priority devoted to strategies regarding social inequalities in RTIs, we believe that a greater emphasis should be put on a few key factors when planning an agenda to confront this problem. These include: (1) the monitoring - that is, the systematic and periodic collection, analysis, and dissemination - of epidemiological data on the impact of RTIs by socioeconomic position; (2) the identification of factors accounting for the observed differences, either due to traffic exposure differences or in risk factors among individuals or communities; (3) the selection of intervention strategies tailored to modify specific factors accounting for social differences; and (4) the appropriate application and evaluation of such strategies.

The development of the equity dimension within the Haddon matrix approach allows for the identification of pre-crash preventive strategies aimed at the individual, such as educational materials and programmes with culturally and linguistically appropriate messages for the specific audiences for whom they are intended-that is, socioeconomically disadvantaged groups. ${ }^{6}$ Similarly, interventions on the physical environment, such as traffic calming and pedestrian protection initiatives, should take into consideration geographical areas and communities where crashes involving the least favoured population groups are more prevalent. As regards factors of the social environment, strategies such as the promotion of community development to increase the ability of socially deprived groups to work together to identify and take action around RTI priorities, participating on decision making in issues like urban renewal and public transportation, should also be considered. ${ }^{7}$ Finally, post-crash strategies should include improving access to emergency and acute care and to rehabilitation services to the whole population, aiming at the reduction of differences in the observed health outcomes among socioeconomic groups. 
In addition, it is advisable to keep a global view of the issue of inequalities in RTIs. This requires the acknowledgement of the concept of "sustainable development", which reminds us that economic development puts pressure on scarce resources, such as energy. As the transfer of existing patterns of car use among Western countries to all people in developing countries is increasingly a burden to global sustainability, more emphasis needs to be placed on health policies promoting high quality public transportation, as well as on the encouragement of walking and cycling as alternatives to car and motorcycle riding. ${ }^{89}$

In other words, curbing the differential impact of RTIs among socioeconomic groups requires a broader use of a set of tools and steps that are available, although researchers and policy makers need to further pursue their efforts in their formulation and use. Otherwise, we are faced with the risk of applying public health strategies in an undiscriminating manner, therefore promoting some form of "socially iatrogenic" policy resulting in a potential enlargement in the negative gap in the health status among socioeconomic groups. In the same way that car and motorcycle makers have been taking great effort in tailoring the sales campaigns of their products to specific social and age subgroups of the population, it is time for the public health community at large to tackle socioeconomic inequities in RTIs in a more systematic and effective way. Although it is generally accepted that the benefits of road transportation are widespread among social groups, it should be none the less a priority to ensure that its resulting risks for health are also evenly distributed in the society.

ANTONI PLASÈNCIA

Institut Municipal de Salut Pública, Barcelona, and Universitat

Autònoma de Barcelona, Spain

CARME BORRELL

Institut Municipal de Salut Pública, Barcelona, Spain

Correspondence to: Dr Plasència, Institut Municipal de Salut Pública Barcelona, Lesseps 1, 08023 Barcelona, Spain

(aplasenc@imsb.bcn.es)

1 Murray CJL, Lopez AD. Alternative visions of the future: projecting mortliaty and disability, 1990-2020. In: Murray CJL, Lopez AD, eds. The global burden of disease. Vol 1. Geneva: World Health Organization, 1996:325-95.

2 Hasselberg M, Laflamme L, Ringbäck Weitoft G. Socioeconomic differences in road traffic injuries during childhood and youth: a closer look at different kinds of road user. $\mathcal{F}$ Epidemiol Community Health 2001;55;858-

3 van

van de Water HPA, van Herten LM. Health policies on target? Leiden: TNO Prevention and Health, Public Health Division, 1998.

4 Haddon W. Options for the prevention of motor vehicle crash injury. Isr $\mathcal{F}$ Med Sci 1980;16:45-65.

5 Runyan CW. Using the Haddon matrix: introducing the third dimension Injury Prevention 1998;4:302-7

6 Agran PF, Winn DG, Anderson CL, et al. Family, social and cultural factors in pedestrian injuries among Hispanic children. Injury Prevention 1998;4:188-93.

7 Whitehead M. Tackling inequalities: a review of policy initiatives. In: Benzeval M, Judge K, Whitehead M. Tackling inequalities in health. An agenda for action. London: King's Fund, 1995:22-52.

8 McCarthy M. Transport and health. In: Marmot M, Wilkinson R. Social determinants of health. New York: Oxford University Press, 1999:13254

9 Independent Inquiry into Inequalities in Health Report. (Chaired by Sir Donald Acheson). London: Stationery Office; 1998.

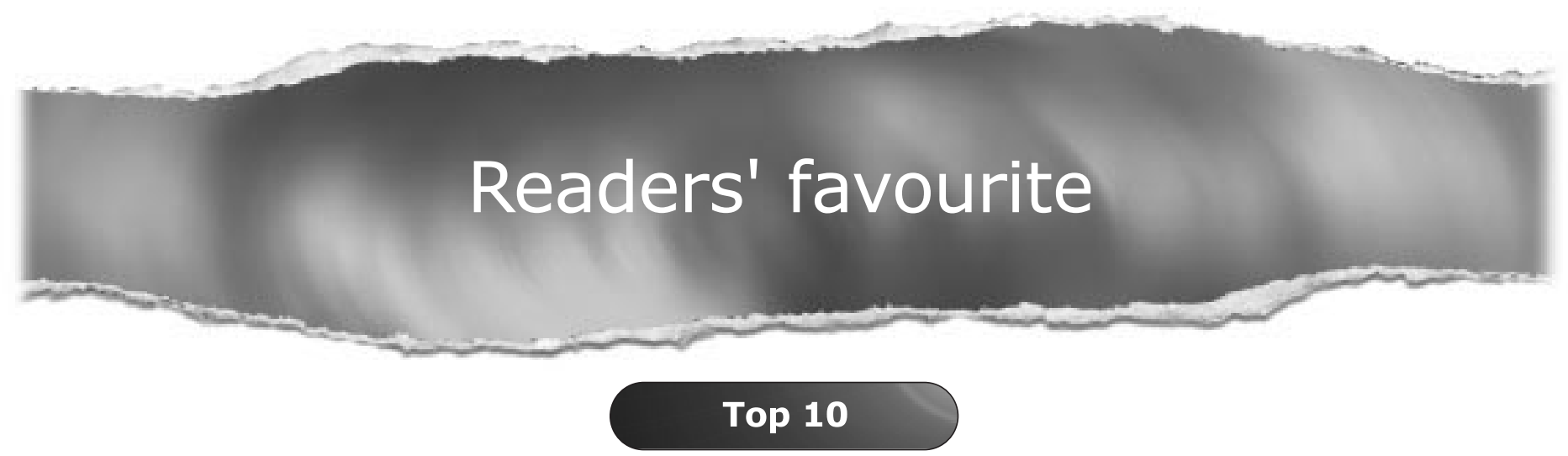

Click on the "Top 10" button on the homepage to see which are the best read articles each month

www.jech.com 